\title{
On the Impossibility, in Some Cases, of the Leray-Hopf Condition for Energy Estimates
}

\author{
John G. Heywood
}

\begin{abstract}
Current proofs of time independent energy bounds for solutions of the time dependent Navier-Stokes equations, and of bounds for the Dirichlet norms of steady solutions, are dependent upon the construction of an extension of the prescribed boundary values into the domain that satisfies the inequality (1.1) below, for a value of $\kappa$ less than the kinematic viscosity. It is known from the papers of Leray ( $\mathrm{J}$ Math Pure Appl 12:1-82, 1993), Hopf (Math Ann 117:764-775, 1941) and Finn (Acta Math 105:197-244, 1961) that such a construction is always possible if the net flux of the boundary values across each individual component of the boundary is zero. On the other hand, the nonexistence of such an extension, for small values of $\kappa$, has been shown by Takeshita (Pac J Math 157:151-158, 1993) for any two or three-dimensional annular domain, when the boundary values have a net inflow toward the origin across each component of the boundary. Here, we prove a similar result for boundary values that have a net outflow away from the origin across each component of the boundary. The proof utilizes a class of test functions that can detect and measure deformation. It appears likely that much of our reasoning can be applied to other multiply connected domains.
\end{abstract}

\section{Introduction}

Let $\Omega \subset R^{n}, n=2$ or 3 , be either a bounded domain or an exterior domain, with boundary $\partial \Omega=$ $\Gamma_{1}+\cdots+\Gamma_{m}$ consisting of a finite number of smooth, bounded, connected components. Let $b^{*}$ be a given smooth vector valued function defined on $\partial \Omega$, and let $\kappa$ be a given positive number. Then an important technical construction (when possible) is that of an extension (i.e., continuation) of $b^{*}$ into $\bar{\Omega}$ as a smooth solenoidal vector field $b$ that satisfies

$$
-\int_{\Omega} \phi \cdot \nabla b \cdot \phi \mathrm{d} x \leq \kappa\|\nabla \phi\|^{2}, \quad \text { for all } \phi \in D(\Omega),
$$

where $D(\Omega)=\left\{\phi \in C_{0}^{\infty}(\Omega): \nabla \cdot \phi=0\right\}$ (or symmetrical such functions in certain symmetrical situations; see Fujita [6]). Integrating by parts and using a shorter notation, we prefer to write this as

$$
(\phi \cdot \nabla \phi, b) \leq \kappa\|\nabla \phi\|^{2}, \quad \text { for all } \phi \in D(\Omega) .
$$

We will say that a given domain $\Omega$ and boundary values $b^{*}$ satisfy the 'Leray-Hopf condition' if, for every $\kappa>0$, there exists a corresponding extension $b$ of $b^{*}$ into $\bar{\Omega}$ satisfying (1.2).

It is well known (see Finn [3]) that any smooth boundary values $b^{*}$ can be extended into the domain as a curl, i.e., $b=\operatorname{curl} \psi$, if and only if the net flux of $b^{*}$ across each $\Gamma_{i}$ is zero. If one is given such an extension, then, for any given $\kappa>0$, one can construct a cut-off function $\eta_{\kappa}$ such that the modified extension $b=\operatorname{curl}\left(\eta_{\kappa} \psi\right)$ satisfies (1.2). If the net flux across some component of the boundary is nonzero, the extension of $b^{*}$ as a curl is impossible, and, in fact, for small values of $\kappa$, it seems to be impossible to extend $b^{*}$ in any manner as a function $b$ satisfying (1.2). We tend to think it is impossible. Impossibility has been proven, by Takeshita [4], in the case of an annular domain and boundary values $b^{*}$ with an inward coming net flux. We give a similar result here, for the case of an outgoing net flux. Both of these results for annular domains easily generalize to domains that contain an annular domain, provided the inner bounding circle or sphere of the annulus encloses one or more components of $\partial \Omega$ from which there is a net inflow or outflow due to $b^{*}$ (which must then pass through the annular domain). 
I am grateful to Professor G. P. Galdi for bringing to my attention his observation (see Section VIII.4 of [5]) that Takeshita's argument in [4] applies only to the case of an incoming net flow, and for requesting my proof in the case of outflow. I had not noticed that Hopf and Takeshita wrote (1.1) and (1.2) with absolute value signs over the left sides. Negation of the inequality is then more easily achieved, by negating either one case or the other, inflow or outflow. Takeshita negated the seemingly easier case for annular domains of inflow.

The proof for outflow given in Sects. $2-5$ is new and somewhat more complicated than the one I previously described to Professor Galdi. Like the earlier proof, it uses what I have called "U-tube test functions". These are introduced in Sect. 2. If one of these test functions is aligned with the diverging flow from a symmetric solenoidal source, the expression on the left of (1.2) will be positive. This is shown in Sect. 3. In Sect. 4 it is shown that the Leray-Hopf condition cannot hold if $\Omega$ is an annular domain bounded by two circles, and the net flux of the boundary values across each bounding circle is directed outwards, away from the origin. This is accomplished by an averaging argument for the existence of a U-tube test function negating (1.2). The corresponding three-dimensional result is given in Sect 5 . These are the main results of the paper.

In Sect. 6 we present our previous simpler proof for the case of outflow. It is based on a symmetrization argument that seems to have little potential for generalization. In Sect. 7 we provide proofs for the case considered by Takeshita, of a net inflow, using test functions that encircle the inner boundary. In Sect. 8 we describe the generalization of the results for annular domains to domains that contain annular domains.

The first result of Sect. 9 is a proposition showing that U-tube test functions provide a natural means of detecting and measuring deformation. This suggests that they should be useful in treating either inflow or outflow. That is shown in a second proposition, where it is found that only very minor modifications in the arguments of Sects. 3-5 are needed to treat the case of inflow. We conclude with our reasons for expecting a more general result.

\section{U-tube Test Functions}

We will describe test functions intuitively, as the velocity fields of a fluid. If $\phi$ is the velocity of a fluid, then $\phi \cdot \nabla \phi$ is (at any point) the directional derivative of the velocity in the direction of the flow, times the speed of the flow, i.e., the acceleration of a particle following a streamline. Thus, in Poiseuille flow through a straight section of tubing, $\phi \cdot \nabla \phi=0$. If a section of such tubing is bent around into a circle, along with each of its geometric streamlines, and the speed of the flow maintained along streamlines to remain solenoidal, then $\phi \cdot \nabla \phi$ will be at every point a nonzero vector directed towards the center of the circular streamline on which the point is located. All streamlines have the same center in the two-dimensional case. In the three-dimensional case their centers lie on a short segment (of length equal to the diameter of the tubing) of the common axis of the circular streamlines.

We construct special test functions $\phi$ as follows. Lay side by side two parallel Poiseuille tubes, left to right and right to left, with some separation between them so that their ends on the right can be joined by a short semi-circular section of tubing, as can their ends on the left. Then we take $\phi$ to be a Poiseuille flow through the straight sections, that continues through the curved end sections by following the geometrically defined streamlines at the same speeds as through the straight sections. Of course, $\phi \cdot \nabla \phi$ will only be nonzero around the curved connecting sections at the ends, where its integral at each end will be a vector pointing back towards the other end. These two vectors are of equal magnitude, which we can assume to be equal to one by adjusting the net flux through the tube. For its U shaped ends, we will refer to $\phi$ as a 'U-tube' test function. Of course it is defined throughout $\Omega$ as zero outside the tubes. While it lacks the smoothness required for membership in $D(\Omega)$, that is a minor issue. If we produce a U-tube test function negating (1.2), so will its mollifications for small averaging radii.

Now consider any two points $\alpha, \beta \in \Omega$ such that the line segment between them, $L=\{\alpha+t(\beta-\alpha)$ : $0 \leq t \leq 1\}$, lies entirely in $\Omega$. We can construct a U-tube test function with straight sections parallel to 
$L$, and with $\alpha$ and $\beta$ at the centers of the semi-circular end sections. Further, we can consider a sequence of these, say $\left\{\phi_{i}\right\}$, with successively smaller tubes and circular radii, so that in Dirac delta style, setting $\eta=(\beta-\alpha) /|\beta-\alpha|$, and letting $F$ be any continuous vector field in $\Omega$,

$$
\left(\phi_{i} \cdot \nabla \phi_{i}, F\right) \rightarrow F(\alpha) \cdot \eta-F(\beta) \cdot \eta, \quad \text { as } i \rightarrow \infty .
$$

\section{Annular $\Omega$ with Symmetric Extensions $b$ of $b^{*}$}

We will treat first the two-dimensional case, and for notational simplicity consider a specific annular domain, $\Omega=\left\{x \in R^{2}: 1 \leq|x| \leq 4\right\}$. As something of a lemma, we begin by considering symmetric boundary values $b^{*}$, equal to the values of $b=\nabla \log |x|$ on the bounding circles, and consider whether $b$ itself satisfies (1.2) for all $\kappa>0$. In fact, it cannot, because taking $\alpha=(2,0)$ and $\beta=(3,0)$, and our test functions $\phi_{i}$,

$$
\left(\phi_{i} \cdot \nabla \phi_{i}, b\right) \rightarrow b(\alpha) \cdot \eta-b(\beta) \cdot \eta, \quad \text { as } i \rightarrow \infty,
$$

and the limit is positive since

$$
b(\alpha) \cdot \eta-b(\beta) \cdot \eta=\left.\frac{\mathrm{d} \log r}{\mathrm{~d} r}\right|_{r=2}-\left.\frac{\mathrm{d} \log r}{\mathrm{~d} r}\right|_{r=3}>0 .
$$

Of course, in choosing a test function we cannot pass to the limit. But we are assured that $\left(\phi_{i} \cdot \nabla \phi_{i}, b\right)>0$ for all large $i$, and choosing one such $\phi_{i}$ denote it by $\varphi$. Then for $\kappa<\kappa_{1} \equiv(\varphi \cdot \nabla \varphi, b) /\|\nabla \varphi\|^{2}$ (still with $b=\nabla \log |x|)$, the inequality (1.2) will not be satisfied by the test function $\varphi$. For future use, set $\lambda \equiv(\varphi \cdot \nabla \varphi, \nabla \log |x|)$.

The three-dimensional case is similar. Let $\Omega=\left\{x \in R^{3}: 1 \leq|x| \leq 4\right\}$ with boundary values $b^{*}$ equal to the values of $b=-\nabla|x|^{-1}$ on the bounding spheres. Then, arguing as before, one sees that $b=-\nabla|x|^{-1}$ does not satisfy (1.2) for a specific three-dimensional U-tube test function $\phi_{i}$, with turning points at $\alpha=(2,0,0)$ and $\beta=(3,0,0)$. We denote this specific $\phi_{i}$ by $\varphi$. Note that since three-dimensional U-tube test functions are not rotationally symmetric about rays from the origin, $\varphi$ must have some particular angle of rotation about the ray through $\alpha$ and $\beta$. But since $b$ is symmetric, this angle is immaterial at this point of the discussion. As before, let $\kappa_{1} \equiv(\varphi \cdot \nabla \varphi, b) /\|\nabla \varphi\|^{2}$, now with $b=-\nabla|x|^{-1}$, and set $\lambda \equiv\left(\varphi \cdot \nabla \varphi,-\nabla|x|^{-1}\right)$.

\section{2-D Annular $\Omega$ with Arbitrary $b^{*}$ and $b$}

Let $\Omega=\left\{x \in R^{2}: 1 \leq|x| \leq 4\right\}$. Let $b=b_{1}+b_{2}$, where $b_{1}=\nabla \log |x|$ and $b_{2}$ is an arbitrary solenoidal function with zero net flux across each component of the boundary, and hence across every circle $|x|=r$ with $r \in[1,4]$. Since $b_{2}$ is arbitrary, we may regard $b^{*}=\left.b\right|_{\partial \Omega}$ as arbitrary (among boundary values with the same net flux across boundary components as $b_{1}$ ) and $b$ as an arbitrary extension of $b^{*}$ into $\Omega$. The test function $\varphi$ we chose above is aligned with the ray of polar angle zero. Let $\varphi_{\omega}$ be its rotation counter clockwise about the origin by the angle $\omega$. Using polar coordinates $r, \theta$ and basis vectors $\widehat{r}(r, \theta), \widehat{\theta}(r, \theta)$, the functions $\varphi$ and $\varphi_{\omega}$ can be expressed as

$$
\begin{aligned}
\varphi(r, \theta) & =\Phi_{1}(r, \theta) \widehat{r}(r, \theta)+\Phi_{2}(r, \theta) \widehat{\theta}(r, \theta) \\
\varphi_{\omega}(r, \theta) & =\Phi_{1}(r, \theta-\omega) \widehat{r}(r, \theta)+\Phi_{2}(r, \theta-\omega) \widehat{\theta}(r, \theta) .
\end{aligned}
$$

From the construction of $\varphi$ it is clear that the dependence of its angular component $\Phi_{2}$ on $\theta$ is symmetric about $\theta=0$, while that of its radial component $\Phi_{1}$ is antisymmetric. Thus, for fixed $\theta$, the dependence of the angular component of $\varphi_{\omega}$ on $\omega$ is symmetric about $\omega=\theta$, and that of its radial component is antisymmetric. Similarly, the radial component of $\varphi \cdot \nabla \varphi$ is symmetric about $\theta=0$, while its angular component is antisymmetric. Thus, for fixed $\theta$, the dependence of the radial component of $\varphi_{\omega} \cdot \nabla \varphi_{\omega}$ on $\omega$ is symmetric about $\omega=\theta$, while that of its angular component is antisymmetric. 
To prove that (1.2) is not satisfied for $\kappa<\kappa_{1}$, it is enough to show that

$$
f(\omega) \equiv \int_{\Omega} \varphi_{\omega} \cdot \nabla \varphi_{\omega} \cdot b \mathrm{~d} x \geq \lambda, \quad \text { for at least one value of } \omega .
$$

For that, it suffices to show that the average of $f(\omega)$ over a period, $0 \leq \omega \leq 2 \pi$, is equal to $\lambda$, i.e.,

$$
\int_{0}^{2 \pi} f(\omega) \mathrm{d} \omega=2 \pi \lambda
$$

Since

$$
f(\omega) \equiv \int_{\Omega} \varphi_{\omega} \cdot \nabla \varphi_{\omega} \cdot\left(b_{1}+b_{2}\right) \mathrm{d} x=\lambda+\int_{\Omega} \varphi_{\omega} \cdot \nabla \varphi_{\omega} \cdot b_{2} \mathrm{~d} x,
$$

this becomes a matter of showing that

$$
\int_{0}^{2 \pi} \int_{\Omega} \varphi_{\omega} \cdot \nabla \varphi_{\omega} \cdot b_{2} \mathrm{~d} x \mathrm{~d} \omega=0 .
$$

This is shown in (4.5) below, where we begin by expressing the integral over $\Omega$ in polar coordinates and changing the order of integration. Then we observe that the symmetry properties of $\varphi_{\omega} \cdot \nabla \varphi_{\omega}$ imply that the integral in square brackets is a normal vector $n(r, \theta)$ to the circle of radius $r$ at $(r, \theta)$. The magnitude of $n(r, \theta)$ depends, of course, only on $r$. The final conclusion is reached remembering that the net flux of $b_{2}$ across every circle $|x|=r$ with $r \in[1,4]$ is zero:

$$
\begin{aligned}
\int_{0}^{2 \pi} \int_{\Omega} \varphi_{\omega} \cdot \nabla \varphi_{\omega} \cdot b_{2} \mathrm{~d} x \mathrm{~d} \omega & \equiv \int_{0}^{2 \pi} \int_{1}^{4} \int_{0}^{2 \pi} \varphi_{\omega}(r, \theta) \cdot \nabla \varphi_{\omega}(r, \theta) \cdot b_{2}(r, \theta) r \mathrm{~d} \theta \mathrm{d} r \mathrm{~d} \omega \\
& =\int_{1}^{4} \int_{0}^{2 \pi}\left[\int_{0}^{2 \pi} \varphi_{\omega}(r, \theta) \cdot \nabla \varphi_{\omega}(r, \theta) \mathrm{d} \omega\right] \cdot b_{2}(r, \theta) r \mathrm{~d} \theta \mathrm{d} r \\
& =\int_{1}^{4}\left(\int_{0}^{2 \pi} n(r, \theta) \cdot b_{2}(r, \theta) r \mathrm{~d} \theta\right) \mathrm{d} r=0 .
\end{aligned}
$$

This completes the proof. It may be possible to reduce the role of symmetry in this argument by viewing the integral (4.5) as an approximation to the difference between the flux integrals across the circles $r=2$ and $r=3$. If we take $\varphi$ to be a thin tube with a small turning radius, then $\varphi_{\omega}(r, \theta) \cdot \nabla \varphi_{\omega}(r, \theta)$ will be zero unless $r$ is just slightly less than 2 or greater than 3 , which are the 'turning regions' of $\varphi_{\omega}$. Hence $n(r, \theta)$ vanishes outside small neighborhoods of the circles $r=2$ and $r=3$.

\section{3-D Annular $\Omega$ with Arbitrary $b^{*}$ and $b$}

Let $\Omega=\left\{x \in R^{3}: 1 \leq|x| \leq 4\right\}$. Let $b=b_{1}+b_{2}$, where $b_{1}=-\nabla|x|^{-1}$ and $b_{2}$ is an arbitrary solenoidal function with zero net flux across each component of the boundary, and hence across every sphere $|x|=r$ with $r \in[1,4]$. Since $b_{2}$ is arbitrary, we may regard the boundary values $b^{*}=\left.b\right|_{\partial \Omega}$ and their extension $b$ as arbitrary. We will use both $\omega$ and $\theta$ to represent position on the unit sphere $S_{1}$, and $d \omega$ and $d \theta$ to denote the corresponding elements of surface area. Let $\varphi_{\omega, \varsigma}$ represent $\varphi$ repositioned along the ray through $\omega$ 
and orientated about that ray by the angle $\zeta, 0 \leq \zeta<2 \pi$. To prove that (1.2) is not satisfied for $\kappa<\kappa_{1}$, it is enough to show (remembering Sect. 3) that

$$
f(\omega, \zeta) \equiv \int_{\Omega} \varphi_{\omega, \zeta} \cdot \nabla \varphi_{\omega, \zeta} \cdot b \mathrm{~d} x \geq \lambda, \quad \text { for at least one choice of } \omega, \zeta .
$$

For that, it suffices to show that the average of $f(\omega, \zeta)$, over all rays (determined by $\omega$ ) and orientations (determined by $\zeta$ ) is equal to $\lambda$, i.e.,

$$
\int_{S_{1}} \int_{0}^{2 \pi} f(\omega, \zeta) \mathrm{d} \zeta \mathrm{d} \omega=8 \pi^{2} \lambda
$$

Since

$$
f(\omega, \zeta) \equiv \int_{\Omega} \varphi_{\omega, \zeta} \cdot \nabla \varphi_{\omega, \zeta} \cdot\left(b_{1}+b_{2}\right) \mathrm{d} x=\lambda+\int_{\Omega} \varphi_{\omega, \zeta} \cdot \nabla \varphi_{\omega, \zeta} \cdot b_{2} \mathrm{~d} x
$$

this becomes a matter of showing that

$$
\int_{S_{1}} \int_{0}^{2 \pi} \int_{\Omega} \varphi_{\omega, \zeta} \cdot \nabla \varphi_{\omega, \zeta} \cdot b_{2} \mathrm{~d} x \mathrm{~d} \zeta d \omega=0
$$

This is shown in (5.5) below, where we begin by expressing the integral over $\Omega$ in spherical coordinates and changing the order of integration. Then we observe that the symmetry properties of $\varphi_{\omega} \cdot \nabla \varphi_{\omega}$ imply that the integral in square brackets is a normal vector $n(r, \theta)$ to the sphere of radius $r$ at $(r, \theta)$. Indeed, for fixed $r$ and $\theta$, the $\omega$ integration may be done in spherical surface coordinates with $\theta$ as pole (i.e., in circles about the ray through $\theta$ ), and $\zeta$ may be measured counterclockwise from these circles. Then, for every $\omega, \zeta$ for which the integrand is nonzero, it will also be nonzero at the point $\widehat{\omega}, \zeta$, where $\widehat{\omega}$ is on the same circle about $\theta$ as $\omega$, but on the opposite side, rotated from $\omega$ by an angle $\pi$. The normal components of $\varphi_{\omega, \zeta}(r, \theta) \cdot \nabla \varphi_{\omega, \zeta}(r, \theta)$ and $\varphi_{\widehat{\omega}, \zeta}(r, \theta) \cdot \nabla \varphi_{\widehat{\omega}, \zeta}(r, \theta)$ will be equal, but their tangential components will be opposite to each other. Thus integration over $\omega$ and $\zeta$ yields a normal vector $n(r, \theta)$ of a magnitude depending only on $r$. The final conclusion is reached by remembering that the net flux of $b_{2}$ across every sphere $|x|=r$ with $r \in[1,4]$ is zero:

$$
\begin{aligned}
\int_{S_{1}} \int_{0}^{2 \pi} \int_{\Omega} \varphi_{\omega, \zeta} \cdot \nabla \varphi_{\omega, \zeta} \cdot b_{2} \mathrm{~d} x \mathrm{~d} \zeta \mathrm{d} \omega & \equiv \int_{S_{1}} \int_{0}^{2 \pi} \int_{1}^{4} \int_{S_{1}} \varphi_{\omega, \zeta}(r, \theta) \cdot \nabla \varphi_{\omega, \zeta}(r, \theta) \cdot b_{2}(r, \theta) r^{2} \mathrm{~d} \theta \mathrm{d} r \mathrm{~d} \zeta \mathrm{d} \omega \\
& =\int_{1}^{4} \int_{S_{1}}\left[\int_{0}^{2 \pi} \int_{S_{1}} \varphi_{\omega, \zeta}(r, \theta) \cdot \nabla \varphi_{\omega, \zeta}(r, \theta) \mathrm{d} \omega \mathrm{d} \zeta\right] \cdot b_{2}(r, \theta) r^{2} \mathrm{~d} \theta \mathrm{d} r \\
& =\int_{1}^{4}\left(\int_{S_{1}} n(r, \theta) \cdot b_{2}(r, \theta) r^{2} \mathrm{~d} \theta\right) \mathrm{d} r=0 .
\end{aligned}
$$

This completes the proof. As in the 2-dimensional case, the integral in (5.5) can be viewed as an approximation to the difference of two surface flux integrals, a point of view which would seem to have a possibility of generalization without symmetry.

\section{Alternative Proof for Annular Domains with Outflow}

After writing the proceeding sections, I found a simpler proof for the outflow case in my students' notes from a course I taught in 1985. I had seen a preprint of Takeshita's paper in 1983 (10 years before its 
publication). Finding it heavy in differential geometry, I found proofs for myself which I assumed were similar to his. The proof given in my course for the case of two-dimensional outflow proceeds as follows.

Consider an annular domain $\Omega \equiv\left\{x \in R^{2}: R_{1}<|x|<R_{2}\right\}$. Choose a U-tube test function $\varphi$ such that

$$
\lambda \equiv(\varphi \cdot \nabla \varphi, \nabla \log |x|) /\|\nabla \varphi\|^{2}>0,
$$

as was shown to be possible in Sect. 3. Suppose $b$ is a smooth solenoidal function defined in $\bar{\Omega}$ such that

$$
\int_{|x|=R_{i}} b \cdot n \mathrm{~d} S=\int_{|x|=R_{i}}(\nabla \log |x|) \cdot n \mathrm{~d} S, \quad \text { for } i=1,2,
$$

and suppose further (seeking to show it leads to a contradiction) that

$$
(\phi \cdot \nabla \phi, b) \leq \kappa\|\nabla \phi\|^{2}, \quad \text { for all } \phi \in D(\Omega), \text { and some } \kappa<\lambda .
$$

To prove this is impossible, we will construct a symmetrization of $b$ using its rotations $b_{\omega}$ about the origin by an angle $\omega$, and similar rotations of its reflection $\widetilde{b}$. Writing $b$ using polar coordinates $r, \theta$ and basis vectors $\widehat{r}(r, \theta), \widehat{\theta}(r, \theta)$, these are defined by

$$
\begin{aligned}
b(r, \theta) & =B_{1}(r, \theta) \widehat{r}(r, \theta)+B_{2}(r, \theta) \widehat{\theta}(r, \theta) \\
b_{\omega}(r, \theta) & =B_{1}(r, \theta-\omega) \widehat{r}(r, \theta)+B_{2}(r, \theta-\omega) \widehat{\theta}(r, \theta) \\
\widetilde{b}(r, \theta) & =B_{1}(r,-\theta) \widehat{r}(r, \theta)-B_{2}(r,-\theta) \widehat{\theta}(r, \theta) \\
\widetilde{b}_{\omega}(r, \theta) & =B_{1}(r,-\theta-\omega) \widehat{r}(r, \theta)-B_{2}(r,-\theta-\omega) \widehat{\theta}(r, \theta) .
\end{aligned}
$$

It is easily seen that (6.3) implies

$$
\left(\phi \cdot \nabla \phi, b_{\omega}\right)=\left(\phi_{-\omega} \cdot \nabla \phi_{-\omega}, b\right) \leq \kappa\|\nabla \phi\|^{2}, \quad \text { for all } \phi \in D(\Omega),
$$

and

$$
(\phi \cdot \nabla \phi, \widetilde{b})=(\widetilde{\phi} \cdot \nabla \widetilde{\phi}, b) \leq \kappa\|\nabla \phi\|^{2}, \quad \text { for all } \phi \in D(\Omega)
$$

and therefore also

$$
\left(\phi \cdot \nabla \phi, \widetilde{b}_{\omega}\right)=\left(\phi_{-\omega} \cdot \nabla \phi_{-\omega}, \widetilde{b}\right) \leq \kappa\|\nabla \phi\|^{2}, \quad \text { for all } \phi \in D(\Omega) .
$$

Clearly the symmetrization

$$
M b(x) \equiv \frac{1}{4 \pi} \int_{0}^{2 \pi}\left[b_{\omega}(x)+\widetilde{b}_{\omega}(x)\right] \mathrm{d} \omega=\nabla \log |x|
$$

since $\nabla \log |x|$ is the only fully symmetric solenoidal function with the same flux across the boundaries as $M b$. Thus, using (6.1), (6.4) and (6.6) we obtain

$$
\begin{aligned}
\lambda\|\nabla \varphi\|^{2} & =(\varphi \cdot \nabla \varphi, \nabla \log |x|) \\
& =\int_{\Omega} \varphi \cdot \nabla \varphi \cdot \frac{1}{4 \pi} \int_{0}^{2 \pi}\left[b_{\omega}+\widetilde{b}_{\omega}\right] \mathrm{d} \omega \mathrm{d} x \\
& =\frac{1}{4 \pi} \int_{0}^{2 \pi} \int_{\Omega}\left[\varphi \cdot \nabla \varphi \cdot b_{\omega}+\varphi \cdot \nabla \varphi \cdot \widetilde{b}_{\omega}\right] \mathrm{d} x \mathrm{~d} \omega \\
& \leq \frac{1}{4 \pi} \int_{0}^{2 \pi} 2 \kappa\|\nabla \varphi\|^{2} \mathrm{~d} \omega=\kappa\|\nabla \varphi\|^{2} .
\end{aligned}
$$


This implies $\lambda \leq \kappa$, while we assumed $\kappa<\lambda$ in (6.3). We conclude that there is no extension $b$ such that (1.2) holds for $\kappa<\lambda$, and therefore that the Leray-Hopf condition is not satisfied. The corresponding three-dimensional proof is similar.

It seems unlikely that this proof by symmetrization and contradiction can be generalized to arbitrary multiply connected domains.

\section{Annular Domains with Inflow}

It was shown by Takeshita [4] that the Leray-Hopf condition is not satisfied by annular domains $\Omega$ together with boundary values $b^{*}$ that have a net inflow (toward the origin) across each bounding circle or sphere. A proof for the two-dimensional case is easily given without any need of symmetrization. Say $\Omega=\left\{x \in R^{2}: 1<|x|<3\right\}$. Then take as test function a 'Poiseuille flow' $\varphi$ in a thin tube situated along the circle $|x|=2$, say between this circle and a circle $|x|=2-\epsilon$. At every point in the tube, the acceleration vector $\varphi \cdot \nabla \varphi$ will point toward the origin. Let us assume that the magnitude of $\varphi$ is adjusted so that $\int_{\Omega}|\varphi \cdot \nabla \varphi| \mathrm{d} x=4 \pi$. Then, if $F$ is a continuous vector field defined in $\Omega$, we will have

$$
(\varphi \cdot \nabla \varphi, F) \approx \int_{|x|=2} F \cdot n \mathrm{~d} s
$$

where $n$ is the unit normal to the circle, pointing toward the origin. The approximations converge as $\epsilon \rightarrow 0$. Assuming, for simplicity, that the prescribed boundary values $b^{*}$ have the same net influx as $b_{1} \equiv-\nabla \log |x|$, we can write their extension as $b=b_{1}+b_{2}$, where $b_{2}$ is a solenoidal function with zero net flux across every circle about the origin. Clearly

$$
\left(\varphi \cdot \nabla \varphi, b_{2}\right)=0 \quad \text { and } \quad\left(\varphi \cdot \nabla \varphi, b_{1}\right) \approx \int_{|x|=2} b_{1} \cdot n \mathrm{~d} s=\left.4 \pi \frac{\mathrm{d} \log r}{\mathrm{~d} r}\right|_{r=2}>0,
$$

and we can assume the approximation is sufficiently good that $\left(\varphi \cdot \nabla \varphi, b_{1}\right)>0$. Thus $(\varphi \cdot \nabla \varphi, b)=$ $\left(\varphi \cdot \nabla \varphi, b_{1}\right)>0$, and it cannot be made smaller by choosing $b_{2}$ differently. This completes the proof in the two-dimensional case.

In the three-dimensional case, we need a symmetrization similar to that used in Sect. 5 . Let $\Omega=$ $\left\{x \in R^{3}: 1<|x|<3\right\}$. In a familiar manner now, we consider vector fields of the form $b=b_{1}+b_{2}$, where $b_{1}=\nabla|x|^{-1}$ and $b_{2}$ is an arbitrary solenoidal function having zero net flux across any spherical surface about the origin. As a basic test function $\varphi$ we take a Poiseuille flow through a tube situated along a great circle about the sphere $|x|=2$. Let points on this sphere be denoted by $\omega$, and the corresponding surface element by $d \omega$. Let $\varphi_{\omega, \varsigma}$ represent $\varphi$ repositioned to pass through the the point $\omega$, leaving it in a direction represented by the angle $\zeta, 0 \leq \zeta<2 \pi$. To prove that (1.2) is not satisfied for $\kappa<\kappa_{1} \equiv\left(\varphi \cdot \nabla \varphi, b_{1}\right) /\|\nabla \varphi\|^{2}$, all that needs to be shown is that

$$
f(\omega, \zeta) \equiv \int_{\Omega} \varphi_{\omega, \zeta} \cdot \nabla \varphi_{\omega, \zeta} \cdot b \mathrm{~d} x \geq\left(\varphi \cdot \nabla \varphi, b_{1}\right), \quad \text { for at least one choice of } \omega, \zeta .
$$

The argument for that is very similar to the reasoning in Sect. 5 above. In effect, the symmetrization provides an approximation of surface flux integrals over the sphere $|x|=2$, by a sum of volume integrals over circular Poiseuille tubes, with the property of giving the exact answer, zero, when integrating functions like $b_{2}$ that have zero net flux across spheres. In that way the proof is completed.

The inflow case will be treated again in Sect. 9, using U-tube test functions.

\section{Domains That Contain Annular Domains}

The previous results easily serve as lemmas in showing that the Leray-Hopf condition cannot be satisfied if the domain $\Omega$ contains an annular region $A$, the inner boundary of which encloses some part of the 
complement of $\Omega$ from which there is either a net inflow or outflow. We may assume $A$ is centered about the origin and that its closure $\bar{A} \subset \Omega$. In that case the boundary values $b^{*}$ prescribed on $\partial \Omega$ can be extended into $\Omega$ in the form $b=b_{1}+b_{2}$, where the restriction of $b_{1}$ to $A$ is a constant times $\pm \nabla \log |x|$ in the two-dimensional case, and $\pm \nabla|x|^{-1}$ in the three-dimensional case, and the net flux of $b_{2}$ is zero across circles or spheres, as before. It is obvious that all of the arguments we have used can be applied to $A$, yielding the desired result for $\Omega$.

\section{On the Possibility of Generalization}

It seemed natural and necessary to use different types of test functions in treating the cases of inflow and outflow. However, the following proposition suggests otherwise, that U-tube test functions are the natural choice in both cases, and are also likely to be useful in a future argument for general domains with either a net inflow or outflow across a bounded component of the boundary. What is shown is that U-tube test functions provide a natural means of detecting and measuring deformation.

Proposition 9.1. Let $b$ be a smooth (not necessarily solenoidal) vector field defined in a two or threedimensional domain $\Omega$. Then the following three conditions are equivalent

(i) $b$ is the velocity field of a rigid motion,

(ii) $(\phi \cdot \nabla \phi, b)=0$, for all $\phi \in D(\Omega)$,

(iii) $(\phi \cdot \nabla \phi, b)=0$, for all $U$-tube test functions $\phi$ with supports in $\Omega$,

and, moreover, deformation can be measured by the methods of the proof, as in (9.4) below.

First we check that (i) implies (ii). A general rigid motion $b$ consists of a uniform translation plus a rotation. Consequently $\nabla b$ is antisymmetric and hence $(\phi \cdot \nabla \phi, b)=-(\phi \cdot \nabla b, \phi)=0$. Of course, (ii) implies (iii). It remains to show that (iii) implies (i). In a neighborhood of any point (say the origin, to simplify the notation) $b$ can be expressed as the sum of a rigid motion, a deformation, and higher order terms

$$
b(x)=b(0)+\left.\Omega b\right|_{0} \cdot x+\left.D b\right|_{0} \cdot x+O\left(|x|^{2}\right) .
$$

Here, $D b=\frac{1}{2}\left(\nabla b+\nabla b^{T}\right)$ and $\Omega b=\frac{1}{2}\left(\nabla b-\nabla b^{T}\right)$ are the deformation and rotation matrices. It is a well known kinematical theorem that if $D b=0$ at every point of an open connected domain, then $b$ is a rigid motion expressible as

$$
b(x)=b(0)+\left.\Omega b\right|_{0} \cdot x .
$$

To check, for a general velocity field $b$, that $D b$ vanishes at some point, choose an orthogonal coordinate system with this point as origin and coordinate axes aligned with the principal axes of $D b$. In this system the deformational part of the velocity field is of the form $\left.D b\right|_{0} \cdot x=\left(\alpha x_{1}, \beta x_{2}, \gamma x_{3}\right)$, for some $\alpha, \beta, \gamma \in R$. It represents a stretching out or squeezing in along the principal axes according to the signs of $\alpha, \beta, \gamma$. These stretchings and squeezings can be detected and measured by evaluating $\left(\phi \cdot \nabla \phi,\left.D b\right|_{0} \cdot x\right)$ for various U-tube test functions. If $\left\{\phi_{\epsilon, i}\right\}$ is a sequence of U-tube test functions with turning points at $(-\epsilon, 0,0)$ and $(\epsilon, 0,0)$, and successively thinner tubes and smaller turning radii as $i \rightarrow \infty$, as assumed in (2.1), then

$$
\lim _{i \rightarrow \infty}\left(\phi_{\epsilon, i} \cdot \nabla \phi_{\epsilon, i},\left.D b\right|_{0} \cdot x\right)=(\alpha(-\epsilon), 0,0) \cdot(1,0,0)-(\alpha \epsilon, 0,0)(1,0,0)=-2 \alpha \epsilon .
$$

Since

$$
\left(\phi_{\epsilon, i} \cdot \nabla \phi_{\epsilon, i}, b(0)+\left.\Omega b\right|_{0} \cdot x\right)=0
$$

by (ii), and

$$
\lim _{i \rightarrow \infty}\left(\phi_{\epsilon, i} \cdot \nabla \phi_{\epsilon, i}, O\left(|x|^{2}\right)\right)=O\left(\epsilon^{2}\right),
$$

we conclude from (9.3) and the continuity of $D b$ that

$$
\alpha=\lim _{\epsilon \rightarrow 0}\left[-\frac{1}{2 \epsilon} \lim _{i \rightarrow \infty}\left(\phi_{\epsilon, i} \cdot \nabla \phi_{\epsilon, i}, b\right)\right] .
$$


The values of $\beta$ and $\gamma$ can be determined similarly. Thus, (iii) implies that $\alpha, \beta$ and $\gamma$ are all zero. Hence $D b$ is zero, and $b$ is a rigid motion. This completes the proof.

Proposition 9.2. The arguments of Sects. 3-5 proving that the Leray-Hopf condition is impossible for annular domains with outflow are easily modified to treat the case of inflow, by taking U-tube test functions orthogonal to rays from the origin.

Consider first the two-dimensional case. In modifying Sect. 3, we take $b=-\nabla \log |x|$, and also $\lambda \equiv$ $(\phi \cdot \nabla \phi,-\nabla \log |x|)$. To obtain U-tube test functions $\phi_{i}$ orthogonal to rays, we may take their turning points to be $\alpha=(2,-\sqrt{5})$ and $\beta=(2, \sqrt{5})$. Then $\eta=(0,1)$ and $(3.2)$ becomes

$$
b(\alpha) \cdot \eta-b(\beta) \cdot \eta=-\left.\frac{\mathrm{d} \log r}{\mathrm{~d} r}\right|_{r=3} \frac{(0,-2 \sqrt{5})}{3}>0 .
$$

In Sect. 4, the only change is that we now take $b_{1}=-\nabla \log |x|$. The changes to the three-dimensional case, in Sects. 3 and 5, are similar. There is one small point to consider. The deployment of U-tubes orthogonally to rays introduces an additional angular variable, which can be fixed by requiring that the centers of the two parallel tubes of a U-tube test function both lie on the same ray from the origin. This completes the proof.

In seeking to prove the impossibility of the Leray-Hopf condition more generally, we seek to detect some fixed amount of deformation with U-tube test functions of fixed dimensions. There is deformation in any extension of the boundary values (other than as a rigid motion) even if the net flux across each component of the boundary is zero. But if this deformation can be restricted to a narrow strip around the boundary (as it is in proving the Leray-Hopf condition when there is zero net flux across each component of the boundary) its detection by U-tube test functions requires thin tubes with small turning radii, and consequently large values of $\|\nabla \varphi\|$, causing a failure to negate (1.2). On the other hand, if there is a nonzero net flux across some component of the boundary, it forces deformation out into the open away from the boundary, where it should be detectable by U-tube test functions of fixed dimensions. Of course, we have shown that it can be detected with such test functions in the case of annular domains. As it is hard to imagine a counter example for non-annular domains that cannot be adapted to annular domains, we expect that the results given here will ultimately be proven for general multiply connected domains.

Open Access. This article is distributed under the terms of the Creative Commons Attribution Noncommercial License which permits any noncommercial use, distribution, and reproduction in any medium, provided the original author(s) and source are credited.

\section{References}

[1] Leray, J.: Etude de diverses équations intégrales nonlinear et de quelques problèmes que pose l'hydrodynamique. J. Math. Pure Appl. 12, 1-82 (1993)

[2] Hopf, E.: Ein allgemeiner Endlichkeitssatz der Hydrodynamik. Math. Ann. 117, 764-775 (1941)

[3] Finn, R.: On the steady-state solutions of the Navier-Stokes equations. III. Acta Math. 105, 197-244 (1961)

[4] Takeshita, A.: A remark on Leray's inequality. Pac. J. Math. 157, 151-158 (1993)

[5] Galdi, G.P.: An Introduction to the Mathematical Theory of the Navier-Stokes Equations Volume II. Springer, Berlin (1994)

[6] Fujita, H.: On stationary solutions to Navier-Stokes equations in symmetric plane domains under general out-flow condition. In: Proceedings of International Conference on Navier-Stokes Equations, Theory and Numerical Methods, June 1997, Varenna Italy, Pitman Research Notes in Mathematics, vol. 388, pp. 16-30

John G. Heywood

Department of Mathematics

University of British Columbia

Vancouver, Canada

e-mail: heywood@math.ubc.ca

(accepted: March 19, 2010; published online: June 8, 2010) 